ThE AstrophysiCal JournaL, 357:L17-L20, 1990 July 1

(C) 1990. The American Astronomical Society. All rights reserved. Printed in U.S.A

\title{
SPIN-UP OF A RAPIDLY ROTATING STAR BY ANGULAR MOMENTUM LOSS
}

\author{
Stuart L. Shapiro ${ }^{1}$ and Saul A. Teukolsky ${ }^{1}$ \\ Center for Radiophysics and Space Research, Cornell University \\ AND \\ TAKASHI NAKAMURA \\ National Laboratory for High Energy Physics, Tsukuba, Japan \\ Received 1990 March 16; accepted 1990 April 11
}

\begin{abstract}
Can a rotating star spin up by radiating away its angular momentum? And if so, what can we infer about the properties of such a star? We show that the spin of a rapidly rotating star like a pulsar can indeed increase as it radiates away energy and angular momentum. For this to happen the adiabatic index governing the equation of state must be very close to $4 / 3$ if the star is rotating uniformly. Otherwise the star must be rotating differentially.
\end{abstract}

Subject headings: pulsars - stars: neutron - stars: rotation — stars: white dwarfs

\section{INTRODUCTION}

The spin-down of a rapidly rotating star is qualitatively different from that of a slowly rotating one. A slowly rotating star is essentially spherical and loss of angular momentum $J$ results in a decrease in the angular rotation rate $\Omega$, but no substantial change in the moment of inertia $I$. A rapidly rotating star, however, may be highly oblate, and the loss of angular momentum can result in a significant decrease in $I$. Like a skater who pulls in her arms while spinning, the star can actually spin up while losing angular momentum. Since $\Omega=J / I$, the sign of the rate of change of $\Omega$ clearly depends on the relative rates of decrease of $J$ and $I$.

The energy and angular momentum of a rotating equilibrium star can slowly decrease by such mechanisms as the emission of electromagnetic or gravitational radiation, or of neutrinos. For example, the emission of magnetic dipole radiation is generally accepted as the dominant spin-down mechanism for radio pulsars. For a uniformly rotating equilibrium star that slowly loses energy $E$ and angular momentum $J$ while its baryon rest mass $M$, entropy $S$, and chemical composition remain constant, the changes in $E$ and $J$ are simply related by

$$
d E=\Omega d J .
$$

This is a general result, proved by Ostriker and Gunn (1969) for Newtonian configurations, and extended by Hartle (1970) to relativistic stars. Rewriting equation (1.1) in the form

$$
\frac{d E}{d t}=\frac{1}{(\partial \Omega / \partial J)_{M, S}} \Omega \frac{d \Omega}{d t},
$$

we see that the sign of $d \Omega / d t$ is determined by the sign of $(\partial \Omega / \partial J)_{M, S}$, since the rate of energy loss $d E / d t$ is negative. The derivative $(\partial \Omega / \partial J)_{M, S}$ depends only on the properties of the equilibrium sequence along which the star evolves, and not on the actual energy loss mechanism.

For the sequence of Maclaurin spheroids, it is straightforward to show that $(\partial \Omega / \partial J)_{M, S}$ changes sign from positive to negative for sufficiently rapidly rotating configurations. But Maclaurin spheroids are homogeneous, incompressible, and

\footnotetext{
${ }^{1}$ Departments of Astronomy and Physics, Cornell University.
}

uniformly rotating. Moreover, the sign change occurs at $T /|W|=0.2379$, well beyond the onset of secular instability at $T /|W|=0.1375$. Here $T$ is the rotational kinetic energy and $W$ is the gravitational potential energy of the star. Does a sign change occur for more realistic inhomogeneous configurations governed by a compressible equation of state? And if it does occur, are the corresponding configurations stable?

Finn and Shapiro (1990) modeled rotating stars as homogeneous, uniformly rotating, compressible spheroids whose central pressure is related to the density by a polytropic equation of state. They found that $(\partial \Omega / \partial J)_{M, S}$ becomes negative for polytropic indices sufficiently close to $n=3$ (adiabatic index sufficiently close to $4 / 3$ ). Thus, these stars spin up as they lose angular momentum. Does the same result apply to real (inhomogeneous) uniformly rotating polytropes with $n$ close to 3 ? We know the result holds for $n=0$, the Maclaurin spheroids. Does it apply to values of $n$ intermediate between 0 and 3 ?

\section{ROTATING SEQUENCES OF CONSTANT $M$ AND $S$}

Many authors have calculated the properties of rapidly rotating Newtonian stars in hydrostatic equilibrium (James 1964; Bodenheimer and Ostriker 1973; Hachisu 1986; see Tassoul 1978 for a detailed discussion and extensive set of references). The equations are cast into nondimensional form and solved numerically. James (1964), for example, introduces the following nondimensional quantities for constructing his sequences of uniformly rotating polytropes:

$$
\begin{aligned}
v & \equiv \frac{\Omega^{2}}{2 \pi G \rho_{c}}, \\
\tilde{M}(v) & \equiv \frac{M}{4 \pi \alpha^{3} \rho_{c}}, \\
\tilde{I}(v) & \equiv \frac{I}{\alpha^{5} \rho_{c}},
\end{aligned}
$$

where $G$ is the gravitational constant, $\rho_{c}$ is the central density, and the unit of length is

$$
\alpha=\left[\frac{(n+1) K}{4 \pi G} \rho_{c}^{-1+1 / n}\right]^{1 / 2} \text {. }
$$


Here

$$
K=\frac{P}{\rho^{1+1 / n}}
$$

is the polytropic constant, a measure of the entropy, and $P$ is the pressure. James tabulates nondimensional model parameters for several values of $\boldsymbol{n}$ along sequences of monotonically varying rotation parameter $v$. However, these numerical sequences do not correspond to true evolutionary sequences of stars that lose angular momentum at fixed rest mass and entropy. To convert the tabulated sequences to evolutionary sequences, we introduce an alternative set of nondimensional parameters:

$$
\begin{aligned}
& \Omega^{*} \equiv \frac{\Omega}{\left[2 \pi G \rho_{c}(0)\right]^{1 / 2}}=\left[\frac{\tilde{M}(0)}{\tilde{M}(v)}\right]^{n /(3-n)} v^{1 / 2}, \\
& J^{*} \equiv \frac{J}{M \alpha^{2}(0)\left[2 \pi G \rho_{c}(0)\right]^{1 / 2}}=\frac{\tilde{I}(v) v^{1 / 2}}{4 \pi \tilde{M}(v)}\left[\frac{\tilde{M}(0)}{\tilde{M}(v)}\right]^{(2-n) /(3-n)} .
\end{aligned}
$$

Here the notation (0) denotes the nonrotating spherical member of the sequence with the same value of $M, K$, and $n$. For each value of $n$, uniformly rotating stars which slowly lose $J$ move along a unique equilibrium curve in the $\Omega^{*}-J^{*}$ plane, parameterized by $v$ and given by equations (2.4). Hachisu (1986) uses a different set of nondimensional quantities from James. However, he provides enough tabulated parameters so that we can also convert his numerical sequences into evolutionary sequences of $\Omega^{*}$ versus $J^{*}$.

The $n=0$ Maclaurin sequence in these units is

$$
\begin{aligned}
& \Omega^{*}=\left[\frac{\left(1-e^{2}\right)^{1 / 2}}{e^{3}}\left(3-2 e^{2}\right) \sin ^{-1} e-\frac{3\left(1-e^{2}\right)}{e^{2}}\right]^{1 / 2}, \\
& J^{*}=\frac{12}{5} \frac{1}{\left(1-e^{2}\right)^{1 / 3}} \Omega^{*},
\end{aligned}
$$

where $e$ is the eccentricity of the spheroid, $0 \leq e \leq 1$. In terms of $e$, the ratio $T /|W|$ is given by

$$
\frac{T}{|W|}=\frac{3}{2 e^{2}}\left[1-\frac{e\left(1-e^{2}\right)^{1 / 2}}{\sin ^{-1} e}\right]-1 .
$$

The curve $\Omega^{*}$ versus $J^{*}$ has a turning point at $e=0.9300$, corresponding to $T /|W|=0.2379$, beyond which $d \Omega^{*} / d J^{*}$ is negative.

In Figure 1 we plot $\Omega^{*}$ versus $J^{*}$ for equilibrium sequences of uniformly rotating stars of constant $M$ and $S$ for various values of polytropic index $n$. The $n=0$ curve is given by the Maclaurin equations (2.5). The other curves are determined from the tabulations of James (1964) and Hachisu (1986). Since the nondimensional tabulated functions $\tilde{M}(v)$ and $\tilde{I}(v)$ are smooth and slowly varying as $n \rightarrow 3$, we use their values for $n=3$ to construct the curves $n=2.9, n=2.95$, and $n=2.97$. Most of the variation in the curves comes from the explicit appearance of $n$ in equations (2.4). Note that the limiting case $n=3$ reduces to a single point: for a given mass and entropy, there is a unique equilibrium configuration $\left(\Omega^{*}, J^{*}\right)$. (Recall that in the spherical case $\Omega^{*}=J^{*}=0$, there is a single mass for each entropy constant $K$ when $n=3$.)

Each polytropic sequence is plotted up to the termination point at which the centrifugal force balances the gravitational attraction at the equator. (The termination point for $n=0$ is off the scale of the plot.) There exist additional sequences of

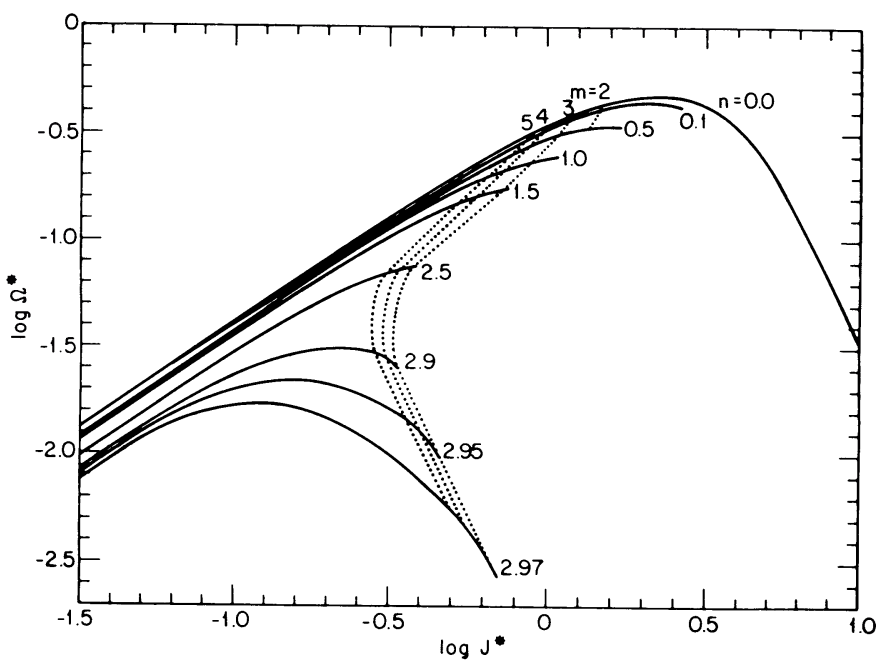

FIG. 1.-Variation of $\Omega^{*}$ vs. $J^{*}$ along uniformly rotating equilibrium sequences of constant $M$ and $S$. The solid lines show the sequences for various values of polytropic index $n$. The dotted lines mark the onset of nonaxisymmetric instabilities of various mode number $m$.

ring and highly flattened, concave-hamburger structures beyond this point (Fukushima et al. 1980; Hachisu 1986), but they do not smoothly join onto the slowly rotating sequence. In addition, they have high values of $T /|W|$ and hence are unstable.

The figure shows the turning point in $\Omega^{*}$ versus $J^{*}$ for the $n=0$ Maclaurin sequence discussed above. It also reveals the existence of a turnover for the nearby sequences $n=0.1,0.5$, and for the sequences with $n$ close to 3 . The latter can be understood by using equations (2.4) to calculate the slope along the equilibrium sequence. For any $n$ we have

$$
\begin{aligned}
& \frac{J}{\Omega}\left(\frac{\partial \Omega}{\partial J}\right)_{M, S}=\frac{d \ln \Omega^{*}}{d \ln J^{*}} \\
& =\left(-\frac{n}{3-n} \frac{d \ln \tilde{M}}{d v}+\frac{1}{2 v}\right) /\left(\frac{d \ln \tilde{I}}{d v}+\frac{2 n-5}{3-n} \frac{d \ln \tilde{M}}{d v}+\frac{1}{2 v}\right) .
\end{aligned}
$$

The limit $n \rightarrow 3$ gives

$$
\frac{d \ln \Omega^{*}}{d \ln J^{*}} \rightarrow-\frac{n}{2 n-5} \rightarrow-3, \quad n \rightarrow 3 .
$$

In fact, for $n \geq 2.5$ all the terms in equation (2.7) are positive except the first term in the numerator. Thus the slope will be negative for a given value of $v$ if $n$ is sufficiently close to 3 . Interestingly, there are no turnovers for values of $n$ well inside the interval $0-3$.

Are any of the models beyond the turning points on the equilibrium curves stable? Several authors have analyzed the stability of uniformly rotating polytropes. Usually only the $m=2$ nonaxisymmetric mode is important (see Tassoul 1978 for a discussion and references), but it is reached only when $n \lesssim 0.808$. For rapidly rotating stars like millisecond pulsars, the modes with $m \lesssim 5$ are relevant, because they are the modes driven unstable by gravitational radiation (Ipser and Lindblom 1989 and references therein). We have used the critical values of $T /|W|$ tabulated by Managan (1985), Imamura, 
Friedman, and Durisen (1985), and Ipser and Lindblom (1990) to plot the curves in Figure 1 at which the configurations become unstable for various modes $m$. For $n$ close to 0 , all models beyond the turning points are clearly unstable. However, for $n$ close to 3 there are models beyond the turning points that are stable. We have therefore demonstrated that if an isolated uniformly rotating star is observed to be spinning up, then it must have an equation of state with $n$ very close to 3 , i.e., adiabatic index $\Gamma$ very close to $4 / 3$.

\section{DIFFERENTIAL ROTATION}

The above conclusion could be altered if the star is undergoing differential rotation. After all, the angular velocity of a satellite in Keplerian orbit about a central mass increases if the satellite loses angular momentum. We thus expect that spin-up of a differentially rotating star losing $J$ might occur under more general circumstances than for a uniformly rotating star.

As confirmation, consider the $n=1.5$ equilibrium models of Hachisu (1986) plotted in Figure 2. The three sequences correspond to three different rotation laws: $u$ for uniform rotation and $v$ and $j$ for two distinct differential rotation laws. The dashed portions of each curve represent toroidal configurations. The spheroidal portion of the $u$ sequence terminates when centrifugal force balances gravitational attraction at the equator. The toroidal portion is not continuously connected to the spheroidal part. The dots mark the points at which $T /|W|=0.14$ on each sequence. The spheroidal portion of the $u$ sequence does not reach this point, while the toroidal part is well beyond this point. The point $T /|W|=0.14$ is the approximate location of the onset of the $m=2$ mode of secular instability for all of these configurations (see, e.g., Ostriker and Bodenheimer 1973; Bardeen et al. 1977). The instability points for the $m>2$ modes have been computed only for the $u$ sequence (see Fig. 1).

As discussed in $\S \mathrm{II}$, the spheroidal portion of the $u$ sequence for $n=1.5$ does not have a turning point where $(\partial \Omega / \partial J)_{M, S}=$ 0 . While the slope of the toroidal portion is negative, it is well

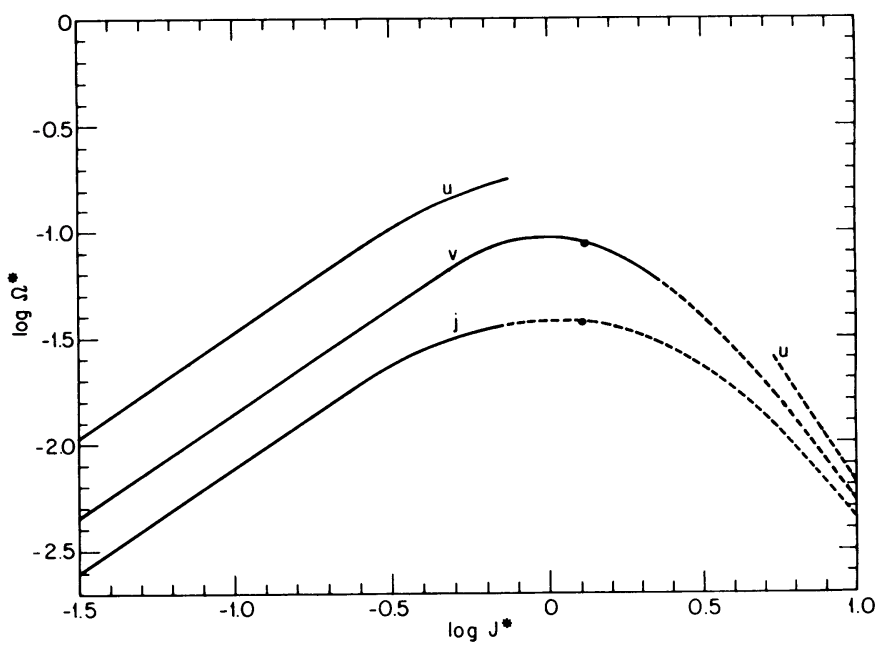

FIG. 2.-Variation of $\Omega^{*}$ vs. $J^{*}$ along several rotating equilibrium sequences of constant $M$ and $S$ for $n=1.5$. The curve labeled $u$ corresponds to uniform rotation, while the curves labeled $v$ and $j$ correspond to the $v$ and $j$ differential rotation laws of Hachisu (1986). The solid portions of each sequence show the spheroidal models, while the dashed portions are toroidal configurations. The solid dots mark the onset of the $m=2$ instability at $T /|W| \approx 0.14$.

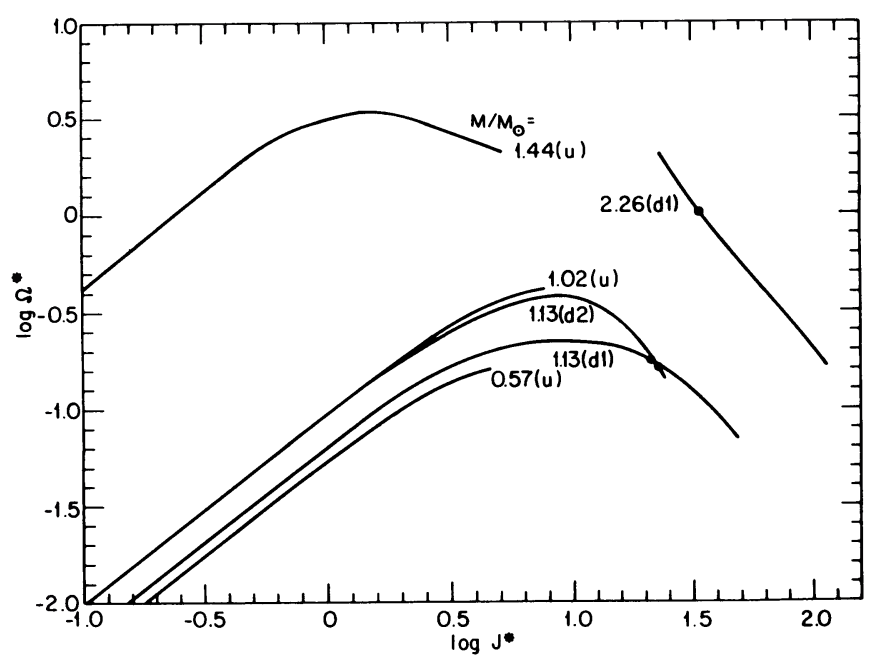

FIG. 3.-Variation of $\Omega^{*}$ vs. $J^{*}$ along several rotating white dwarf sequences. Each curve is labeled by the mass $M$. Curves labeled by $u$ correspond to uniform rotation. Curves labeled by $\mathrm{d} 1$ correspond to the $\boldsymbol{n}^{\prime}=\mathbf{0}$ differential rotation law of Ostriker and Tassoul (1969). Curves labeled by d2 correspond to their $n^{\prime}=3 / 2$ differential rotation law. The solid dots mark the onset of the $m=2$ instability at $T /|W| \approx 0.14$.

beyond the instability point. However, there do exist spheroidal configurations on the $v$ sequence which have negative slopes and are stable to the $m=2$ mode. While these configurations may still be unstable to higher $m$ modes, it nevertheless seems plausible that there may exist stable, differentially rotating stars which can undergo spin-up for $n$ quite different from 3. Such behavior depends crucially on the rotation law; it does not occur along the $j$ sequence. Moreover, an evolving star may move off a particular sequence if the differential rotation law changes. The closer $n$ becomes to 3 , however, the less sensitive is the dependence on the rotation law.

\section{WHITE DWARFS: A NUMERICAL DEMONSTRATION}

The most interesting application of the possibility of spin-up via angular momentum loss is to radio pulsars. However, most of the numerical computations of realistic stars governed either by uniform or differential rotation and by a reliable equation of state have been carried out for white dwarfs (see, e.g., James 1964; Ostriker and Tassoul 1969; Durisen 1975; Hachisu 1986). These detailed models illustrate all of the general conclusions reached above.

For example, in the case of uniform rotation, rapidly rotating stars near the Chandrasekhar limit of $1.4 M_{\odot}$ will spin up as they lose $E$ and $J$. Such stars are supported by relativistically degenerate electrons and so have $n \approx 3$ and are stable. Low-mass white dwarfs, by contrast, will not exhibit this behavior since $n$ is closer to 1.5 . These expectations are borne out in Figure 3, where uniformly rotating white dwarfs of various masses are denoted by a $u$.

By contrast, in the case of differential rotation we expect that even stars of low mass can undergo spin-up. Both differentially rotating models, denoted $\mathrm{d} 1$ and $\mathrm{d} 2$ in the figure, spin up at high $J^{*}$ for $M=1.13 M_{\odot}$, whereas uniformly rotating models with the same mass do not. Note that the turning points on these two sequences occur well before the $m=2$ instability points. With sufficient differential rotation, one can have equilibrium white dwarfs with masses well above the Chandrasekhar limit. The $M=2.26 M_{\odot}$ curve shows such a sequence. It 
has a negative slope everywhere, and some of the models are stable.

\section{v. CONCLUSIONS}

We have shown that rapidly rotating stars can actually spin up while losing angular momentum. If they are in uniform rotation, the equation of state must have an adiabatic index close to 4/3. Differential rotation may allow a larger value of adiabatic index. Nonaccreting millisecond pulsars are the most promising candidates for observing this behavior. These objects are rapidly rotating neutron stars that can lose angular momentum by electromagnetic and gravitational radiation, keeping their baryon mass constant and their entropy essentially zero. When it is detected, the newborn neutron star in $\mathrm{SN}$ 1987A may also be a candidate.

To explore this effect further, it would be most useful if future tabulations of rotating equilibrium models represented sequences of constant $M$ and $S$. Only then would the sequences correspond to evolutionary tracks.

We expect that similar spin-up can occur in rapidly rotating stellar models constructed in general relativity. This question can also be resolved by building equilibrium sequences of fixed baryon mass and entropy.

It is a pleasure to thank R. H. Durisen and L. S. Finn for several helpful discussions. This work has been supported in part by National Science Foundation grants AST 87-14475, PHY 86-03284 and INT 88-15793 at Cornell University. This cooperative research project has been supported jointly by the NSF and JSPS under the US-Japan Cooperative Science Program. Fellowship support from the John Simon Guggenheim Memorial Foundation is gratefully acknowledged by S. L.S.

\section{REFERENCES}

Bardeen, J. M., Friedman, J. L., Schutz, B. F., and Sorkin, R. 1977, Ap. J. (Letters), 217, L49.

Bodenheimer, P., and Ostriker, J. P. 1973, Ap. J., 180, 159.

Durisen, R. H. 1975, Ap. J., $199,179$.

Finn, L. S., and Shapiro, S. L. 1990, Ap. J., in press

Fukushima, T., Eriguchi, Y., Sugimoto, D., and Bisnovatyi-Kogan, G. S. 1980, Progr. Theoret. Phys., 63, 1957.

Hachisu, I. 1986, Ap. J. Suppl., $61,479$.

Hartle, J. B. 1970, Ap. J., 161, 111.

Imamura, J. N., Friedman, J. L., and Durisen, R. H. 1985, Ap. J., $294,474$.

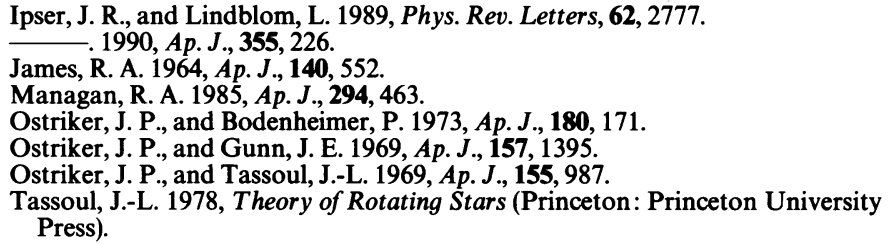

Stuart L. ShapIro and SaUl A. Teukolsky: Space Sciences Building, Cornell University, Ithaca, NY 14853

Takashi Nakamura: National Laboratory for High Energy Physics, Oho, Tsukuba-shi, Ibaraki-ken, 305 Japan 